\title{
DISECCIÓN AXILAR DIRIGIDA UTILIZANDO CARBÓN VEGETAL COMO MARCADOR EN PACIENTE CON CÁNCER DE MAMA TRATADA CON QUIMIOTERAPIA NEOADYUVANTE. TÉCNICA QUIRÚRGICA
}

\author{
JOSÉ NAVARRO MARTÍNEZ' \\ LILIÁN NAVARRO CAMPOS \\ BEXAIDA NAVARRO CAMPOS
}

TARGETED AXILLARY DISSECTION USING CHARCOAL AS A MARIKER IN A PATIENT WITH BREAST CANCER TREATED WITH NEOADJUVANT CHEMOTHERAPY. SURGICAL TECHNIQUE

RESUMEN

Objetivos: Describir la técnica quirúrgica de la disección axilar dirigida (DAD) usando carbón vegetal como marcador del ganglio linfático axilar metastásico al momento del diagnóstico conjuntamente con la biopsia del ganglio centinela en paciente con cáncer de mama tratada con quimioterapia neoadyuvante con respuesta completa clínica y ecográficamente, demostrando su seguridad y eficacia. Material y Métodos: Estudio retrospectivo y descriptivo. Paciente con cáncer de mama y ganglio linfático metastásico en axila ipsilateral marcado con carbón vegetal al confirmarse ese diagnóstico y tratada con quimioterapia neoadyuvante con posterior negativización tanto clínica como ecográfica del ganglio linfático metastásico. Se planificó para disección axilar dirigida (extirpación del ganglio marcado con carbón vegetal y biopsia de ganglio centinela) con el fin de demostrar la eficacia del marcador utilizado y su relación o no con el ganglio centinela. Resultados: Se comprobó la identificación certera del ganglio afectado marcado con carbón vegetal el cual no presentó migración del colorante o reacción inflamatoria local coincidiendo además con dos ganglios centinelas todos con respuesta patológica completa. El carbón permaneció 153 días desde su administración hasta la cirugía axilar. Conclusión: Esta experiencia admite el marcaje con carbón vegetal del ganglio axilar metastásico al momento de su diagnóstico como un método seguro, sencillo, económico y accesible en relación a otros métodos de marcaje, además su asociación con la biopsia del ganglio centinela nos permite prescindir de la disección axilar en caso de respuesta patológica completa, sin embargo, es importante resaltar que se necesitan evaluar más casos para obtener conclusiones determinantes.

Palabras clave: Quimioterapia neoadyuvante; marcaje con carbón; cáncer de mama, disección axilar dirigida; biopsia ganglio centinela; estadificación axilar; disección axilar.

\footnotetext{
1. Cirujano General. Miembro Titular de la Sociedad Venezolana de Cirugía. Miembro Titular de la Sociedad Venezolana de Mastología. Correo-e: josejnavarom@gmail.com

2. Cirujano General. Miembro Asociada de la Sociedad Venezolana de Cirugía. Miembro Asociada de la Sociedad Venezolana de Mastología.
}

\begin{abstract}
Objectives: To describe the surgical technique of targeted axillary dissection using charcoal marking of the metastatic lymph node at the time of diagnosis together with sentinel node biopsy in a breast cancer patient who received neoadjuvant chemotherapy with complete clinical and sonographical response, demonstrating its safety and efficacy. Material and Methods: Retrospective and descriptive study. Patient with breast cancer and metastatic lymph node in the ipsilateral axilla which was marked with charcoal upon confirmation of this diagnosis and treated with neoadjuvant chemotherapy with subsequent clinical and sonographical negativization of the metastatic lymph node. It was planned for targeted axillary dissection (removal of charcoal-marked lymph node and sentinel node biopsy) in order to demonstrate the efficacy of the marker used and its relationship or not with the sentinel node. Results: The correct identification of the affected lymph node marked with charcoal was verified, which did not present dye migration or local inflammatory reaction, also coinciding with two sentinel nodes, all of them with a complete pathological response. The charcoal remained 153 days from its administration until the axillary surgery. Conclusion: This experience supports charcoal marking of the metastatic lymph node at the time of diagnosis as a safe, simple, inexpensive and accessible method in relation to other marking methods, in addition its association with sentinel node biopsy allows us to dispense with axillary dissection in case of complete pathological response, however it is important to highlight that more cases need to be evaluated to obtain decisive conclusions.
\end{abstract}

Key words: Neoadjuvant therapy; charcoal marking; breast cancer; targeted axillary dissection; Sentinel lymph node biopsy; axillary staging; axillary lymph node dissection. 


\section{INTRODUCCIÓN}

La biopsia de ganglio centinela (BGC) en pacientes con cáncer de mama con axila clínica y ecográficamente negativa es el método de elección en la estadificación axilar demostrando ser una técnica precisa y con una tasa de falsos negativos aceptables "

La disección axilar (DA) en pacientes con axila positiva, confirmada por anatomía patológica, representa el tratamiento estándar, sin embargo, la administración de quimioterapia neoadyuvante (OTNA) en pacientes con cáncer de mama y metástasis ganglionar axilar que presentan una respuesta excelente, manifestado por la negativización desde el punto de vista clínico y ecográfico, representa actualmente un tema de controversia, ya que en presencia de una respuesta patológica completa (RPC) comprobada mediante el marcaje de este ganglio metastásico (GM) al momento de su diagnóstico y que posteriormente es extirpado en conjunto con la BGC significaría que muchas pacientes podrían beneficiarse de una mínima intervención que omitiría la realización de una DA y por ende la aparición de sus complicaciones entre ellas el linfedema. ${ }^{(2)}$

La disección axilar dirigida (DAD), en pacientes que han recibido QTNA, representa la combinación de la extirpación del ganglio axilar metastásico, confirmado por anatomía patológica, marcado con semilla de yodo radiactivo y la BGC. En caso de una respuesta patológica completa de este ganglio marcado y del ganglio centinela esto reemplazaría la DA. Actualmente el procedimiento de DAD es considerado un método muy preciso en la estadificación axilar post QTNA en vista de sus bajas tasas de falsos negativos. ${ }^{(3,4)}$

\section{MATERIAL Y MÉTODOS}

Presentamos el caso de una paciente con cáncer de mama tratada con OTNA con repuesta clínica y ecográfica completa en la axila, a quien se le practicó DAD, utilizando carbón vegetal como marcador del GM al momento de su diagnóstico, también se realizó BGC con el fin de evaluar la seguridad y eficacia del carbón vegetal como marcador de este tipo de lesiones, así como su relación con la $\mathrm{BGC}$

Paciente femenina, 63 años, quien consultó por nódulo palpable en mama izquierda. Antecedentes: Menarquía: 15 años. Edad de primer embarazo a término: 25 años. Número de embarazos: 2 Gestas. 2 Paras. Edad de menopausia: 55 años. Terapia de reemplazo hormonal: Niega. Tabáquicos: Niega. Antecedentes personales de enfermedades mamarias: Condición fibroquística de la mama. Antecedentes familiares de enfermedades mamarias prima materna con cáncer de mama. Cirugía mamaria previa: Niega. Antecedente de PAAF de mama izquierda de quiste simple.

Examen físico: discreta retracción de piel en el cuadrante ínfero externo de la mama izquierda y se palpa nódulo de bordes imprecisos. Axila izquierda clínicamente positiva: ganglio axilar pequeño firme y muy móvil.

Mamografía: mamas heterogéneamente densas tipo C concluida como BIRADS 0 sin imágenes especificas descritas. Ultrasonido mamario: se identificó lesión sólida, heterogénea, hipoecoica, no paralela, forma irregular, márgenes no circunscritos, algunos angulares, altamente sospechosa en radial 4, BIRADS 5 ecográfico, coincidiendo con nódulo palpable de 1,5 x 1,2 cm. Axila izquierda: Ganglio linfático hipoecoico, hilio excéntrico con engrosamiento de la corteza altamente sospechoso de $1,5 \mathrm{~cm}$. (Figura 1)

Biopsia con aguja gruesa de 14 G ecoguiada de la lesión en mama izquierda informó carcinoma ductal infiltrante, moderadamente diferenciado, grado nuclear 2 y bajo índice mitótico y citología de ganglio linfático sospechoso axilar mediante punción - aspiración con aguja fina (PAAF) eco guiada informó carcinoma ductal metastásico. (Figura 2).

Inmunohistoquímica: RE positivo 100\%, RP positivo 10\%, Her/2 neu positivo, Ki 67: 5\%. Se le realizaron estudios de extensión mediante tomografía de tórax, abdomen y pelvis sin evidencia de enfermedad a distancia, correspondiendo a un estadio IB.

\section{Técnica del marcaje del GM con carbón vegetal}

El procedimiento es realizado por un cirujano general con más de 20 años de dedicación a la mastología y con entrenamiento en ecografía mamaria e intervencionismo. Se realiza de forma individual y presencial. En el consultorio se coloca a la paciente en decúbito supino con brazo izquierdo en abducción y la mano localizada detrás de la cabeza con apoyo en región escapular.

Se localiza ecográficamente el GM, se realiza asepsia de la piel de la región axilar, se coloca un cobertor en el transductor, se administra 1 cc de Lidocaína al 1\% en piel y subcutáneo, se
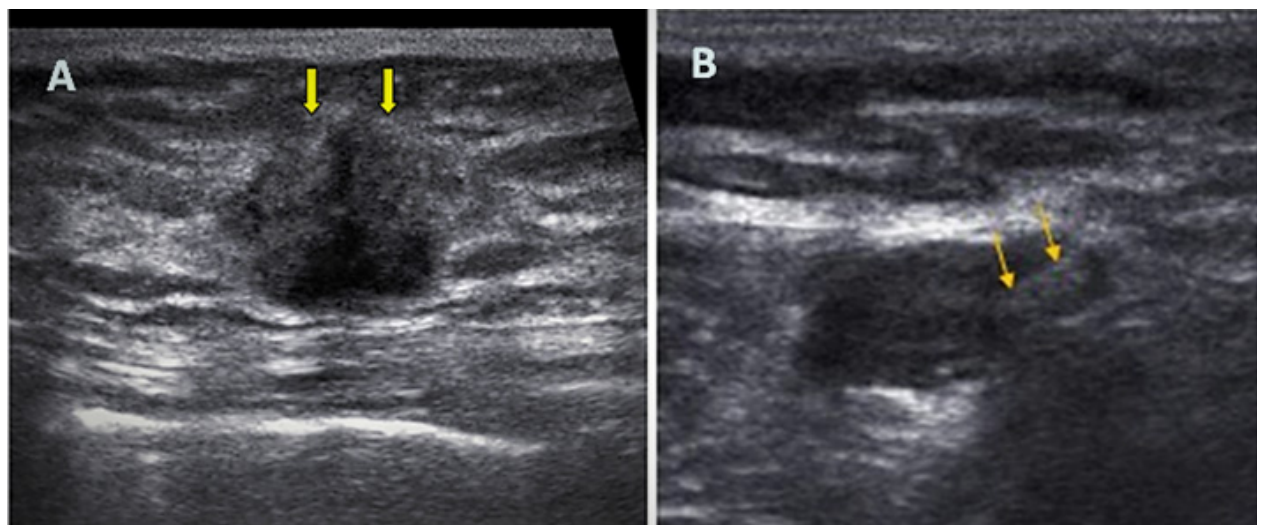

Figura. 1. A) Lesion soirda altamente sospechosa al uitrasonido mamario (BIRAADS 5), Iocalizado en cuadrante infero externo de mama izquierda, coincidiendo con anomalia palpable de 1,3 $1,2 \mathrm{~cm}$. B) Imagen ultrasonografica de ganglio linfatico sospechosode 1,5 cm. de diametro mayor Nótese area muy hipoecoica de aspecto nodular hilio excentrico poco visible el cual se encuentra en el extremo derecho de la imagen (area ecogenica) señalado con flechas amarilla. 

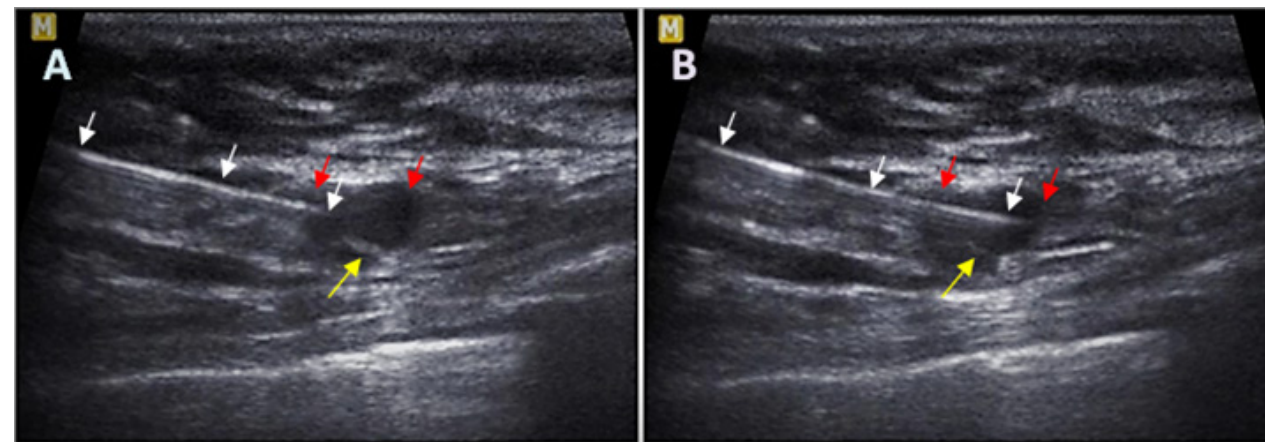

Figura 2. Imagenes de PAAF eco guiada de ganglio axilar sospechoso en axila. A) Posicionamiento de aguja fina, 23 G, (flechas blancas) en ganglio linfático (flechas rojas). Se identifica hilio ganglionar excéntrico (flecha amarilla). B) Incursión de aguja en el interior de ganglio afectado mientras se aplica vacio a la inyectadora para obtener material para estudio citopatológico (flechas: similar a lo anteriormente descrito)

La cirugía se efectuó 153 días después del marcaje con carbón vegetal del ganglio metastásico y de la lesión mamaria

Técnica quirúrgica de la DAD

En quirófano, bajo anestesia general inhalatoria, se inicia el procedimiento con la inyección subareolar del Azul Patente® (azul de disulfina 2,5\% p/v) en plano subdérmico.

Se practica abordaje de la procede con aguja fina de $23 \mathrm{G}$ - 1 1/2 conectada a jeringa con 1 cc de carbón vegetal (Negro Site®) comercializado en Venezuela por el grupo Soluven 2011 C.A del laboratorio Adalgor C.A. Se introduce aguja bajo guía ecográfica hasta un punto circundante periganglionar cenital (superficial), nunca en el interior del ganglio. se procede a administrar el carbón lentamente observando cambios ecográficos en tiempo real que consisten en formación de una imagen ecogénica con desplazamiento del ganglio hacia el plano profundo (Figura 3). Similar procedimiento se realiza en la lesión mamaria. Este procedimiento será realizado una sola vez previo a la QTNA

Recibe posteriormente OTNA: Docetaxel, Doxorubicina y Ciclofosfamida (TAC) cada 21 días por 6 ciclos y la evaluación al finalizar el tratamiento demostró negativización clínica y ultrasonográfica de la axila, la lesión mamaria no era palpable observándose imagen ultrasonográfica residual de 1,2 × 0,6 cm.

Se planificó para tumorectomía, DAD (extirpación de ganglio afectado marcado con carbón y BGC) con evaluación patológica transoperatoria de márgenes de tumorectomía, ganglio marcado y ganglio centinela.

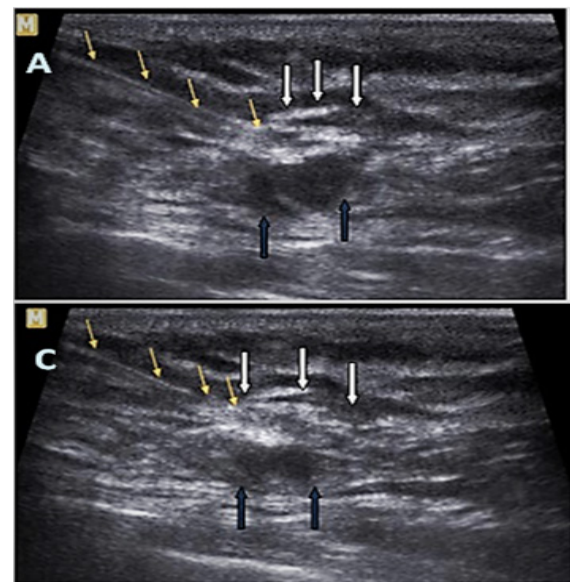

axila 15 minutos posteriores a la inyección del azul patente. Se realiza incisión arciforme, diéresis por planos hasta llegar a la fascia axilar la cual se escinde en la misma dirección de la incisión de piel. Se identifica el tatuaje de carbón y estructuras teñidas de azul compatible con vasos linfáticos y ganglio centinela (Figura 4 A y B) se procede con disección roma en el plano subfascial identificando el ganglio centinela (tenido de azul) y el ganglio marcado (carbón vegetal) evitando la tripsia directa a los tejidos teñidos, se procede a extirpar en bloque toda el área de tejido teñido con tejido celulo adiposo circundante La evidencia macroscópica nos hace inferir coincidencia del ganglio tatuado con carbón vegetal con el ganglio centinela (Figura 4 C y D). El informe transoperatorio del patólogo confirmó que el ganglio marcado coincidió con el ganglio centinela. Se identificaron dos ganglios centinelas que se informaron como negativos para metástasis. Posteriormente en segundo tiempo quirúrgico se procede a la realización de la tumorectomía, realizando extirpación de la lesión con márgenes que se confirman por anatomía patológica como negativos.

La biopsia definitiva confirmó una respuesta patológica completa en los dos ganglios centinelas con tamaño de 0,4 cm

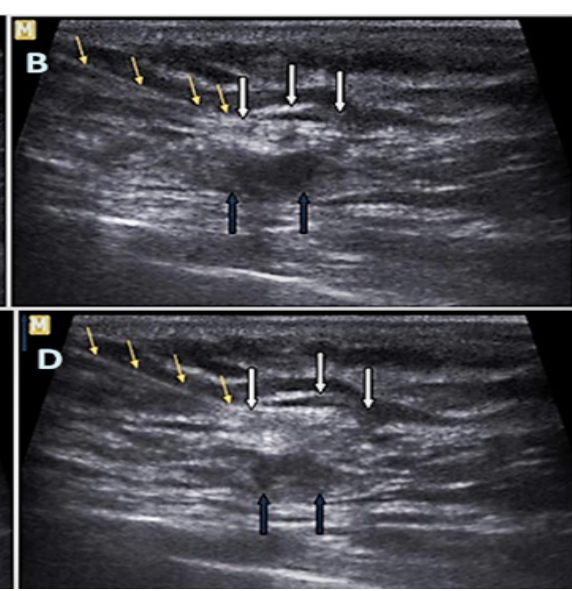

Figura 3. Secuencia de administración de carbón vegetal (1 cc.) guiado por ultrasonido de ganglio linfático metastasico en axila A) Posicionamiento de aguja fina 23 G (flechas amarillas) en tejido circundante, peri ganglionar, en un punto cenital en relación al ganglio (flechas blancas), el borde inferior del ganglio se señala con flechas azules. B) Se observa formación de imagen ecogénica en parte superior de ganglio metastásico al administrar el carbón con discreto desplazamiento del mismo en sentido inferior. C) Aumento de la imagen peri ganglionar superior por efecto de infiltración del carbón. D) Al finalizar la administración del carbón la imagen ecogénica, cenital en relación al ganglio, se hace más prominente con mayor desplazamiento del ganglio en sentido inferior 


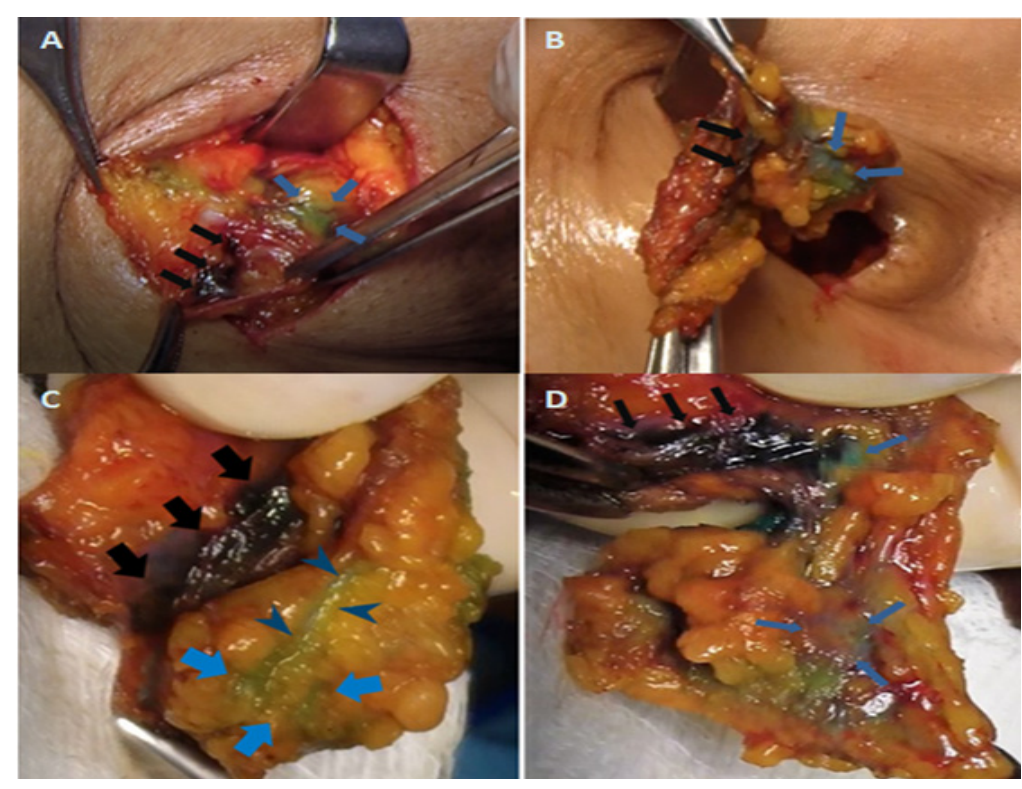

igura 4. secuencia de fotos de DAD, GM marcado con carbon y BGC. A) dentificación transoperatoria de tatuaje de carbón vegetal (flechas negras ganglio centinela teñido de azul (flechas azules) B) Reconocimiento de ganglio centinela (flechas azules) y adyacente se observa rastro de carbón vegetal (flechas negras). C) Imagen de DAD realizada sugiriendo coincidencia entre ganglio metastásico marcado con carbón y ganglio centinela. Flechas negras; marcaje con carbón. Flechas azules: ganglio centinela rodeado de tejido adiposo, Cabeza de flechas azules: vaso linfático. D) Pieza quirúrgica de DAD: tatuado con carbón (flechas negras) y ganglio centinela (flechas azules).

cada uno y lesión residual en cáncer de mama de 2 × 3 mm. La paciente evolucionó satisfactoriamente y se programa para radioterapia

Se obtuvo el consentimiento informado de la paciente incluida en este estudio. Los procedimientos fueron realizados como fueron planificados sin modificaciones.

\section{DIscusıón}

Existen numerosos marcadores que se pueden utilizar: clips de acero, titanio, hidrogel o ácido poliglicólico, semillas de lodo 125, radiofrecuencia o ferromagnéticas que se dejan a través de una punción en el interior del GM de manera ecoguiada y que posteriormente utilizan diferentes métodos de localización y recuperación (arpón, sonda de detección gamma, magnéticas o radiofrecuencia) cuando se realiza la cirugía axilar después de QTNA. Estos métodos necesitan equipos costosos y personal entrenado en materiales y dispositivos de manejo complejo especialmente el material radiactivo. ${ }^{15}$

Experiencia reciente en la utilización de carbón vegetal como marcador del GM axilar, comprobado por anatomía patológica, demostró una gran precisión en su extirpación posterior a la QTNA y sin interferencia con la BGC en la misma intervención informando de una identificación de GM marcado entre 93,6 y 100\%, coincidencia del ganglio marcado con GC entre 60 y $98,5 \%$. La permanencia del carbón fue entre 21 y 148 días. ${ }^{(6,7,8)}$

Evaluando los resultados de estos trabajos constatamos que la DAD utilizando carbón en el marcaje del GM o sospechoso antes de la OTNA presenta unos excelentes resultados y si a esto le sumamos la sencillez del procedimiento, prácticamente sin complicaciones y bajo costo representaría una técnica de marcaje de gran valor a tener en cuenta para la DAD especialmente en países o centros con limitados recursos tecnológicos

Las últimas guías europeas y norteamericanas publicadas comienzan a protocolizar el manejo de las pacientes que presentan RPC después de la OTNA con el objetivo de reducir la magnitud de la cirugía en la axila y evitar la DA. $(9,10)$

Indudablemente se tendría que esperar por los resultados de estudios multicéntricos y estandarizados para ofrecer a cada paciente un tratamiento oncológico seguro basado en las características clínicas, moleculares, respuesta al tratamiento y en concordancia con las variables de estadificación axilar post OTNA entre las cuales la DAD, por ahora, tiene alta relevancia.

\section{CONFLICTOS DE INTERESES}

Este trabajo no fue financiado por ninguna persona natural o jurídica. Los autores declaran que no tienen ningún conflicto de intereses.

\section{REFERENCIAS}

1. Mansel RE, Fallowfield L, Kissin M, Goyal A, Newcombe RG, Dixon JM, et al. Randomized multicenter trial of sentinel node biopsy versus standard axillary treatment in operable breast cancer: the ALMANAC trial. J Natl Cancer Inst. 2006 May;98(9):599-609. https://pubmed. ncbi.nlm.nih.gov/16670385/

2. Riogi B, Sripadam R, Barker D, Harris O, Innes H, Chagla L. Management of the axilla following neoadjuvant chemotherapy for breast cancerA change in practice. Surgeon. 2021 Feb; 19(1):1-7. https://pubmed. ncbi.nlm.nih.gov/32192932/

3. Caudle A, Yang W, Krishnamurthy S, Mittendorf E, Black D, Gilcrease M, et al. Improved Axillary Evaluation Following Neoadjuvant Therapy for Patients with Node-Positive Breast Cancer Using Selective Evaluation of Clipped Nodes: Implementation of Targeted Axillary Dissection. J Clin Oncol. 2016 Apr;34(10):1072-78. https://pubmed.ncbi.nlm. nih.gov/26811528/

4. Simons J, Van Nijnatten T, Van der Pol C, Luiten E, Koppert L, Smidt M. Diagnostic Accuracy of Different Surgical Procedures for Axillary Staging After Neoadjuvant Systemic Therapy in Node-positive Breast Cancer. A Systematic Review and Meta-analysis. Annals of Surgery. 2019 March;269(3):432-42. https://pubmed.ncbi.nlm.nih. gov/30312200/

5. Flores D, Aguilar J, Martínez M, Ibáñez M, Carrasco L, et al. El problema de la estadificación axilar en el cáncer de mama tras quimioterapia neoadyuvante. Papel de la disección axilar dirigida y tipos de marcadores ganglionares. Cir Esp. 2020 Nov;98(9):51 0-15.https:// medes.com/publication/156850

6. Hartmann S, Kuhn T, Boniface J, Stachs A, Winckelmann A, Frisell J et 
al. Carbon tattoing for targeted lymph node biopsy after primary systemic therapy in breast cancer: prospective multicentre TATTOO trial. BJS. 2021 March; 108(3):302-07. https://doi.org/10.1093/bjs/ znaa083

7. Natsiopoulos I, Stavros I, Liappis T, Zarampoukas K, Zarampoukas T, Zacharopoulou V, et al. Axillary lymph Node Tattooing and Targeted Axillary Dissection in Breast Cancer Patients Who Presented as $\mathrm{cN}+$ Before Neoadjuvant Chemotherapy and Became cNO After Treatment. Clin Breast Cancer. 2019 Jun; 19(3):208-15. https:// pubmed.ncbi.nlm.nih.gov/30922804/

8. Park S, Koo J, Kim G, Sohn J, Kim S, Cho Y et al. Feasibility of Charcoal Tattooing of Cytology-Proven Metastatic Axillary Lymph Node at Diagnosis and Sentinel Lymph Node Biopsy after Neoadjuvant Chemotherapy in Breast Cancer Patients. Cancer Res Treat. 2018 Jul;50(3):80 1-12. https://www.ncbi.nlm.nih.gov/pmc/articles/ PMC6056962/
9. Franceschini G. Sentinel node biopsy after neoadjuvant chemotherapy for breast cancer in patients with pretreatment node-positive: Recommendations to optimize the performance. Eur J Surg Oncol. 2020 Jan;46(1):216-17. https://pubmed.ncbi.nlm.nih gov/31676199/

10. Gandhi A, Coles C, Makris A, Provenzano E, Goyal A, Maxwel A et al. Axillary Surgery Following Neoadjuvant Chemotherapy Multidisciplinary Guidance from the Association of Breast Surgery, Faculty of Clinical Oncology of the Royal College of Radiologists, UK Breast Cancer Group, National Coordinating Committee for Breast Pathology and British Society of Breast Radiology. Clin Oncol. 2019 Sep;3 19):664-68. https://pubmed.ncbi.nlm.nih.gov/31229382/ 